\title{
Efficient and cost-effective bacterial mRNA sequencing from low input samples through ribosomal RNA depletion
}

\author{
Chatarin Wangsanuwat ${ }^{1,2+}$, Kellie A. Heom ${ }^{1,2+}$, Estella Liu', Michelle A. O'Malley ${ }^{1,2}$ and Siddharth S. Dey ${ }^{1,23^{*}}$ (D)
}

\begin{abstract}
Background: RNA sequencing is a powerful approach to quantify the genome-wide distribution of mRNA molecules in a population to gain deeper understanding of cellular functions and phenotypes. However, unlike eukaryotic cells, mRNA sequencing of bacterial samples is more challenging due to the absence of a poly-A tail that typically enables efficient capture and enrichment of mRNA from the abundant rRNA molecules in a cell. Moreover, bacterial cells frequently contain 100-fold lower quantities of RNA compared to mammalian cells, which further complicates mRNA sequencing from non-cultivable and non-model bacterial species. To overcome these limitations, we report EMBR-seq (Enrichment of mRNA by Blocked rRNA), a method that efficiently depletes 5S, 165 and 23 S rRNA using blocking primers to prevent their amplification.

Results: EMBR-seq results in 90\% of the sequenced RNA molecules from an E. coli culture deriving from mRNA. We demonstrate that this increased efficiency provides a deeper view of the transcriptome without introducing technical amplification-induced biases. Moreover, compared to recent methods that employ a large array of oligonucleotides to deplete rRNA, EMBR-seq uses a single or a few oligonucleotides per rRNA, thereby making this new technology significantly more costeffective, especially when applied to varied bacterial species. Finally, compared to existing commercial kits for bacterial rRNA depletion, we show that EMBR-seq can be used to successfully quantify the transcriptome from more than 500-fold lower starting total RNA.
\end{abstract}

Conclusions: EMBR-seq provides an efficient and cost-effective approach to quantify global gene expression profiles from low input bacterial samples.

Keywords: Bacterial mRNA sequencing, mRNA enrichment, rRNA depletion, Low input total RNA

\section{Background}

Bacterial species pervade our biosphere and millions of years of evolution have optimized these microbes to perform specific biochemical reactions and functions; processes that could potentially be adapted to develop a variety of products, such as renewable

\footnotetext{
* Correspondence: sdey@ucsb.edu

${ }^{+}$Chatarin Wangsanuwat and Kellie A. Heom contributed equally to this work. 'Department of Chemical Engineering, University of California Santa Barbara, Santa Barbara, CA 93106, USA

${ }^{2}$ Center for Bioengineering, University of California Santa Barbara, Santa Barbara, CA 93106, USA

Full list of author information is available at the end of the article
}

biofuels, antibiotics, and other value-added chemicals [1-5]. Bacterial messenger RNA (mRNA) sequencing provides a snapshot of the genome-wide state of a microbial population, and therefore enables fundamental understanding of these varied microbial functions and phenotypes [6].

However, compared to eukaryotes, mRNA sequencing from bacterial samples has been more challenging for several reasons. First, unlike in eukaryotes, bacterial mRNA does not contain a poly-A tail at the $3^{\prime}$ end that can be used to easily enrich for these molecules during reverse transcription [7, 8]. Further, total RNA isolated from bacterial cells typically contains greater than 95\%

\section{$\triangle B M C$}

(c) The Author(s). 2020 Open Access This article is licensed under a Creative Commons Attribution 4.0 International License, which permits use, sharing, adaptation, distribution and reproduction in any medium or format, as long as you give appropriate credit to the original author(s) and the source, provide a link to the Creative Commons licence, and indicate if changes were made. The images or other third party material in this article are included in the article's Creative Commons licence, unless indicated otherwise in a credit line to the material. If material is not included in the article's Creative Commons licence and your intended use is not permitted by statutory regulation or exceeds the permitted use, you will need to obtain permission directly from the copyright holder. To view a copy of this licence, visit http://creativecommons.org/licenses/by/4.0/ The Creative Commons Public Domain Dedication waiver (http://creativecommons.org/publicdomain/zero/1.0/) applies to the data made available in this article, unless otherwise stated in a credit line to the data. 
ribosomal RNA (rRNA), and therefore cost-effective and high coverage sequencing of the transcriptome requires the development of efficient strategies to deplete the abundant 5S, 16S and 23S rRNA molecules [9]. Finally, bacterial cells typically contain approximately 100-fold lower RNA than mammalian cells, and as the starting amount of total RNA when working with rare, noncultivable, and non-model bacterial species can be limiting, it is a challenge to robustly and accurately capture the transcriptome from small quantities of total RNA with minimal amplification biases [10].

Several commercial kits have been developed to deplete bacterial rRNA from total RNA samples, including the MICROBExpress Bacterial mRNA Enrichment Kit (Thermo Fisher Scientific), the RiboMinus Transcriptome Isolation Kit, bacteria (Thermo Fisher Scientific), and the Ribo-Zero rRNA Depletion Kit (Illumina) [11]. These techniques rely on subtractive hybridization to deplete rRNA and typically work at a scale of hundreds of nanograms to micrograms of starting total RNA. Further, as these commercial kits are only effective on species targeted in the standard probe set, it is challenging to extrapolate these methods to diverse bacterial species $[9,11]$. While this limitation of pre-designed kits has been overcome through the development of workflows to generate custom subtractive hybridization probe sets for any species of interest, they still operate at microgram quantities of starting material and either require multiple rounds of hybridization or a series of oligo optimization steps prior to optimal performance [12, 13]. An alternate approach relies on the Terminator ${ }^{\mathrm{Tn}} 5^{\prime}$ phosphate-dependent exonuclease (TEX) (Lucigen) to specifically degrade rRNAs with $5^{\prime}$-monophosphate ends but not mRNAs with 5 '-triphosphate ends; however, this method typically has lower efficiencies than other existing rRNA depletion strategies [10, 14, 15]. A more recent method uses complementary single-stranded DNA probes to tile rRNAs that are subsequently degraded by RNase H [16]. The commercial NEBNext Bacteria rRNA depletion kit (NEB) employs a similar strategy and can be applied to as low as $10 \mathrm{ng}$ of starting total RNA. Similarly, another approach uses a pool of tiled single-guide RNAs to direct Cas9 mediated cleavage of rRNA-derived cDNA to deplete rRNA while another approach uses targeted reverse transcription primers designed to avoid capturing rRNAs $[17,18]$. However, all these methods require a large array of probes that can be expensive to synthesize and potentially need to be redesigned for distant bacterial species [16-18].

Therefore, in this work we have developed EMBR-seq (Enrichment of mRNA by Blocked rRNA), a new technology that overcomes the limitations of sequencing mRNA from bacterial samples by: (1) Using 5S, $16 \mathrm{~S}$ and 23S rRNA blocking primers and poly-A tailing to specifically deplete rRNA and enrich mRNA during downstream amplification; (2) Using a single or a few blocking primers for each of the three abundant rRNA molecules, thereby enabling rapid adaptation to different bacterial species and significantly reducing the cost per sample; and (3) Using a linear amplification strategy to amplify mRNA from as low as 20 picograms of total RNA with minimal amplification biases. We applied EMBR-seq to a model E. coli system to demonstrate efficient mRNA enrichment and sequencing with increased sensitivity in gene detection. Further, we show that our method accurately captures the genome-wide gene expression profiles with minimal technical biases. Thus, EMBR-seq is an efficient and cost-effective approach to sequence mRNA from low-input bacterial samples.

\section{Results \\ EMBR-seq uses blocking primers to deplete rRNA}

To overcome the limitations described above, we developed EMBR-seq, a new technique to efficiently deplete rRNA from total RNA, thereby enabling cost-effective sequencing of mRNA from bacterial cells. To minimize rRNA-derived molecules in the final sequencing library, we first incubated the total RNA with rRNA blocking primers, designed specifically to bind the $3^{\prime}$ end of $5 \mathrm{~S}$, $16 \mathrm{~S}$ and $23 \mathrm{~S}$ rRNA, followed by poly-adenylation with $E$. coli poly-A polymerase (Fig. 1 and Methods). To deplete rRNA, EMBR-seq only requires primers at the 3 ' end of rRNA, unlike recent methods that tile oligonucleotides along the entire length of rRNA molecules, thereby significantly reducing costs and making our approach more easily translatable to other bacterial species. The blocking primers generate double-stranded RNA-DNA hybrid molecules at the $3^{\prime}$ end of rRNAs, which reduces subsequent poly-adenylation and downstream amplification of rRNA molecules, as the poly-A polymerase preferentially adds adenines to single-stranded RNA [19]. Thereafter, the reaction mixture is reverse transcribed following the addition of a poly- $T$ primer. This primer has an overhang containing a samplespecific barcode to enable rapid multiplexing and reduction in library preparation costs, the $5^{\prime}$ Illumina adapter, and a T7 promoter [20]. After second strand synthesis, cDNA molecules are amplified by in vitro transcription (IVT). However, as only cDNA molecules deriving from a poly-adenylated RNA have a T7 promoter, our technique further amplifies mRNAderived molecules for sequencing whereas rRNAderived molecules are excluded from IVT amplification. The amplified RNA from IVT is then used to prepare Illumina sequencing libraries, as described previously (Fig. 1 and Methods) [20-22]. 


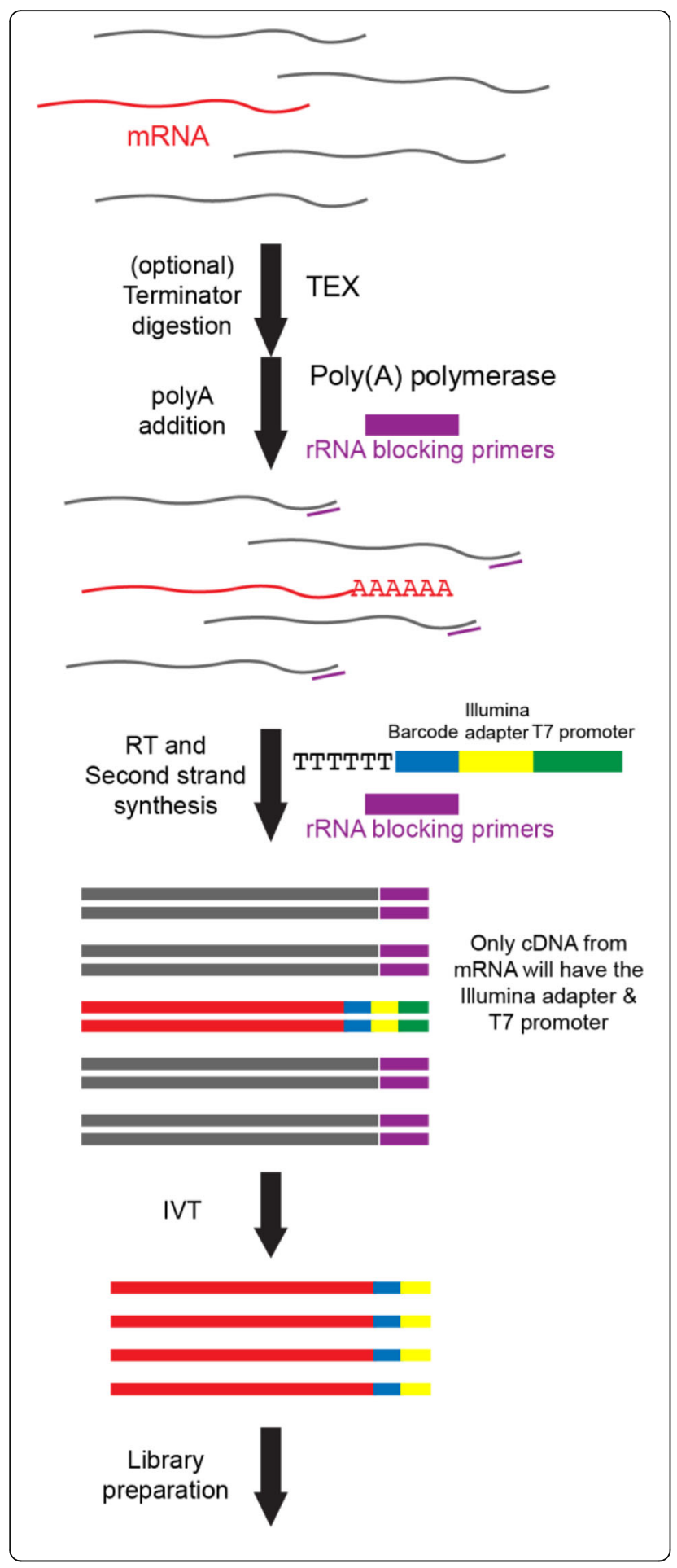

Fig. 1 Schematic of EMBR-Seq. After performing an optional Terminator $^{\text {TM }} 5$ '-phosphate-dependent exonuclease digestion, poly-A polymerase and rRNA blocking primers (purple) are added to total bacterial RNA (mRNA in red and rRNA in gray). Blocking primers specifically bind to the $3^{\prime}$ end of $5 S, 16 S$, and 235 rRNAs, resulting in the preferential addition of a poly-A tail to mRNA molecules. Next, reverse transcription is performed using (i) a poly-T primer, which has an overhang containing a sample-specific barcode (blue), 5' Illumina adapter (yellow), and T7 promoter (green), and (ii) rRNA blocking primers to convert poly-adenylated RNA and rRNA molecules, respectively, to cDNA. The cDNA molecules are then amplified by in vitro transcription, and the amplified RNA is used to prepare Illumina libraries. As the rRNA-derived CDNA does not contain a T7 promoter, these molecules are not amplified during in vitro transcription, resulting in rRNA depletion

\section{EMBR-seq efficiently depletes rRNA to sequence bacterial mRNA}

We applied EMBR-seq to total RNA isolated from the exponential growth phase of E. coli strain K12 (MG1655). Starting from $100 \mathrm{ng}$ of total RNA, we were able to successfully make Illumina libraries that were sequenced and mapped to the E. coli transcriptome. In parallel, we prepared control libraries where total RNA was processed using the EMBR-seq protocol but in the absence of blocking primers. While total RNA from $E$. coli has previously been reported to consist of $95 \%$ rRNA [9], our control samples with no blocking primers had approximately $64 \% \mathrm{rRNA}$, consistent with previous observations that mRNA molecules are preferentially poly-adenylated compared to rRNA even in the absence of any blocking primers (Fig. 2a) [23, 24]. Importantly, compared to the control samples, we observed a significant increase in rRNA depletion efficiency (from 64 to $16 \%$ ), with $84 \%$ of the mapped reads corresponding to mRNA in samples treated with blocking primers (Fig. 2a). As tRNAs make up another major class of RNA molecules, we analyzed our data to quantify the detection of these molecules [25]. We found tRNA-derived reads to constitute only 0.37 and $1.26 \%$ of the mapped reads in the control and EMBR-seq samples, respectively. This expected low detection rate is likely due to the small size of tRNAs, their stable secondary structures and utilization of numerous post-transcriptionally modified nucleotides that are known to interfere with reverse transcription [26, 27]. Overall, these results demonstrate that EMBR-seq achieves a level of mRNA enrichment that is better or comparable to recent bacterial rRNA depletion reports [11-13, 15-18].

In certain applications, such as those where RNA is extracted from non-cultivable bacterial species within natural isolates, the total RNA can be fragmented and of poor quality. To determine if EMBR-seq can still be successfully applied to degraded RNA, we compared the rRNA depletion efficiency of total E. coli RNA with two 

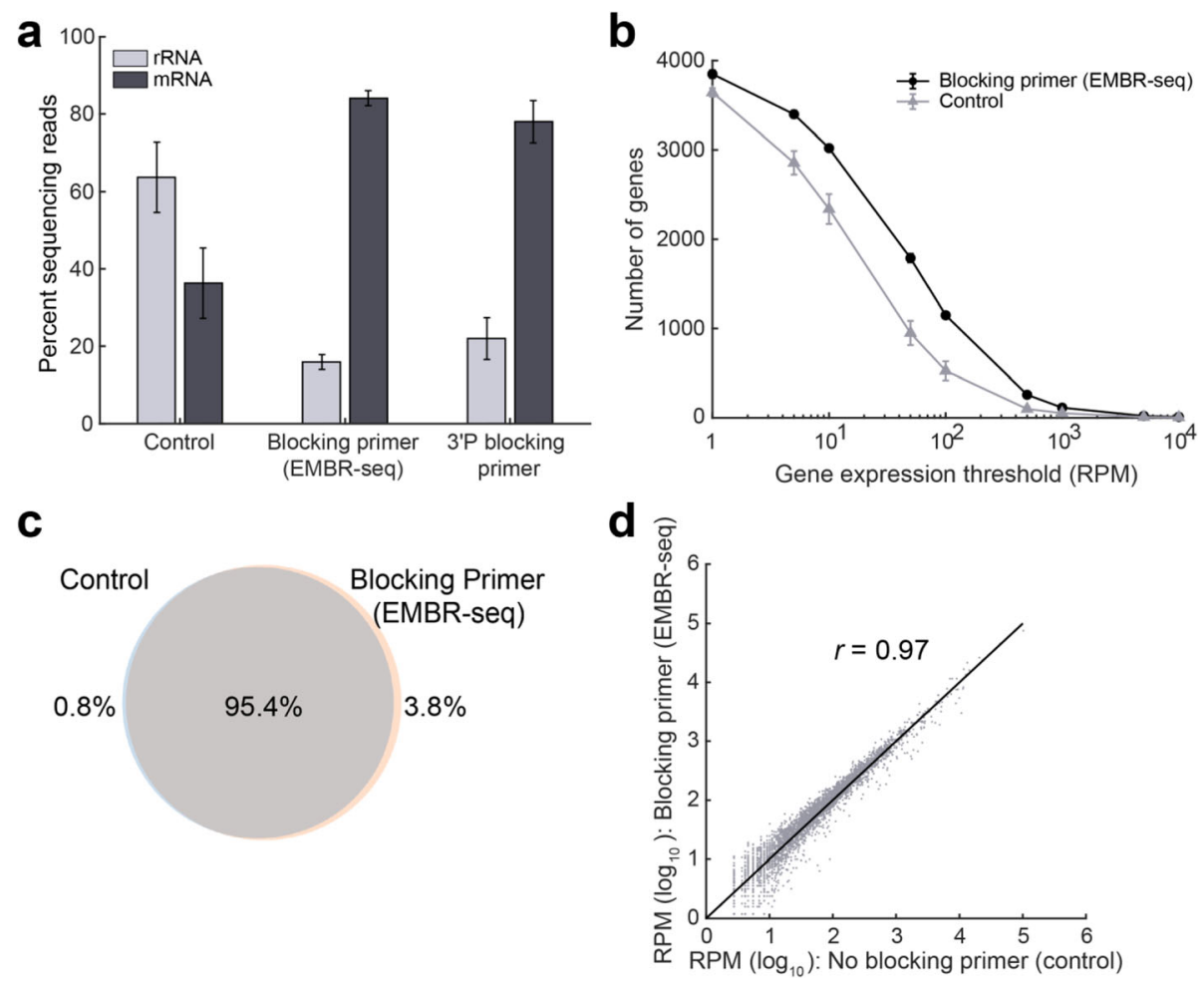

Fig. 2 Blocking primers in EMBR-seq deplete rRNA and provide a deeper view of the transcriptome without introducing technical biases. a In the presence of blocking primers, a 4-fold rRNA depletion and more than 2-fold mRNA enrichment is achieved compared to control samples. With the introduction of blocking primers in EMBR-seq, mRNAs account for more than $80 \%$ of the mapped reads, which is a greater than 16 -fold increase compared to total RNA in E. coli cells. The 3' phosphorylated blocking primers display similar but slightly lesser mRNA enrichment $(n \geq 2$ replicates for all conditions). $\mathbf{b}$ Comparison between EMBR-seq and control samples in the number of genes detected above different expression thresholds ( $n=3$ for both conditions). For the EMBR-seq group, error bars are of the same scale as the size of the data points. $\mathbf{c}$ Venn diagram shows that more than 99\% of the genes detected in the control samples were also detected when using blocking primers in EMBR-seq. 99.2\% of all detected genes were found in the EMBR-seq samples and $96.2 \%$ in the control samples. The number of genes detected were calculated by combining data obtained from three control samples and three EMBR-seq processed samples. $\mathbf{d}$ Gene transcript counts with and without blocking primers are highly correlated (Pearson $r=0.97$ ) suggesting that EMBR-seq does not introduce technical artifacts in quantifying gene expression ( $n=3$ for both datasets). These experiments were performed starting with $100 \mathrm{ng}$ total RNA from E. coli. Error bars in panels (a) and (b) represent standard deviations

different RIN (RNA Integrity Number) scores of 7.2 and 2.4. While RNA molecules can be fragmented by several mechanisms, the degraded sample with a RIN score of 2.4 in this experiment was prepared by heating the total RNA at $95^{\circ} \mathrm{C}$ for $5 \mathrm{~min}$. Surprisingly, we observed that EMBRseq depleted rRNA to similar levels of 15 and $17 \%$ in the untreated and degraded samples, respectively (Additional file 1: Fig. S1). We hypothesize this occurs because both rRNA and mRNA molecules are fragmented to similar extents, and the increased poly-adenylation of rRNA fragments is matched by a similar increase in polyadenylation of mRNA fragments, resulting in similar downstream detection of rRNA- and mRNA-derived reads. Thus, these results suggest that EMBR-seq can be effectively applied to sequence the transcriptome of randomly fragmented lower quality total RNA.

We also tested modified blocking primers with a 3' phosphorylation, designed to prevent Superscript II from reverse transcribing rRNA molecules. As expected, we observed rRNA depletion in these samples as well (from 64 to $22 \%$ ), with $78 \%$ of the mapped reads corresponding to mRNA (Fig. 2a). However, compared to the unmodified blocking primers, these phosphorylated blocking primers were slightly less efficient at rRNA depletion (Fig. 2a). As the 3' phosphorylated primers prevent polymerase extension, we hypothesize that the reduced rRNA depletion efficiency arises from the small fraction of rRNA molecules that get poly-adenylated, primed by the poly-T primers, and copied through the short $30 \mathrm{bp}$ RNA-DNA hybrid due to the strand-displacement activity of the reverse transcriptase. Therefore, given the reduced efficiency and higher costs of the 3 ' phosphorylated blocking primers, all further experiments were performed with unmodified blocking primers.

As an alternate strategy, we also incorporated TEX treatment in EMBR-seq as it has previously been shown to specifically degrade rRNAs with $5^{\prime}$-monophosphate ends but not mRNAs that have $5^{\prime}$-triphosphate ends 
$[10,14,15,28]$. While we again observed rRNA depletion and a corresponding enrichment of mRNA compared to control samples, the effects were less pronounced with a less than 2-fold rRNA depletion, consistent with previous reports (Additional file 1: Fig. S2) [14, 15]. We hypothesize that this reduced efficiency may arise from the additional cleanup step that is necessary prior to treatment with the poly-A polymerase. As a result, we find that blocking primers alone provide the most significant rRNA depletion and mRNA enrichment, and therefore all further experiments were performed without TEX treatment.

\section{EMBR-seq is a cost-effective bacterial mRNA sequencing technology}

In designing the steps of EMBR-seq, we wanted to develop a method that is both easily applied and costeffective. Due to its simplicity, the cost per rRNA depletion reaction in EMBR-seq is $\sim \$ 0.40$, which is at least an order of magnitude lower than other recent rRNA depletion methods and commercial kits [11-13, 15-18] (Additional file 1: Fig. S3a, Table S1, and Additional file 2). The total cost of EMBR-seq, starting from total bacterial RNA to the final Illumina library, was estimated to be $\sim$ \$36 per sample. However, the total cost per sample decreases as more samples are multiplexed in the same Illumina library. For example, when 96 samples are multiplexed, the cost per sample drops to $\sim \$ 20$, primarily due to the pooling of samples after second-strand synthesis that then requires only a single IVT and Illumina library preparation reaction downstream (Additional file 1: Fig. S3b and Additional file 2). Thus, EMBR-seq is a simple and cost-effective approach to sequence mRNA from total bacterial RNA.

\section{EMBR-seq provides a detailed view of the transcriptome without introducing technical biases}

Next, we systematically compared the gene expression profiles obtained from control and rRNA depleted samples to investigate if the use of blocking primers provides a deeper view of the transcriptome without introducing technical artifacts. First, after downsampling sequencing reads to the same depth, we detected 3628 genes in the control samples, while in the mRNA enriched samples we detected 3852 genes, with $99 \%$ of the genes in the control samples also detected in the mRNA enriched samples (Fig. 2b, c). Moreover, at different levels of downsampling, we detected more genes using EMBRseq compared to the control samples (Additional file 1: Fig. S4). This suggests that we can measure the genomewide gene expression landscape in a more cost-effective way using EMBR-seq. Further, the number of genes detected above different expression thresholds was consistently higher for the mRNA enriched samples compared to the control samples (Fig. 2b). This shows that EMBR- seq is able to detect more genes at different gene expression levels, spanning over three orders of magnitude. Furthermore, we also observed that EMBR-seq derived reads mapped uniformly across the entire length of operons, with modest $3^{\prime}$ and $5^{\prime}$ end bias, suggesting that this method can be used to effectively quantify the expression of genes within operons (Additional file 1: Fig. S5). Finally, we observed that gene expression between the control and mRNA enriched samples were highly correlated (Pearson $r=0.97$ ) revealing that the blocking primers do not introduce technical biases in the quantification of gene expression (Fig. 2d). Collectively, these results demonstrate that our new cost-effective method is able to accurately capture the transcriptome of bacterial cells.

\section{EMBR-seq allows mRNA sequencing from low input total RNA}

In many practical applications involving non-model and non-cultivable bacterial species, the starting amount of total RNA available for RNA sequencing can be limiting. Therefore, we evaluated if we can successfully deplete rRNA and quantify gene expression from lower amounts of input material. We applied EMBR-seq to 20, 2, 0.2 and $0.02 \mathrm{ng}$ of starting total RNA isolated from the exponential growth phase of E. coli strain K12. These starting quantities of total RNA were chosen as they are typically below the sensitivity and detection limit of commercial kits and previously reported methods [11, 17]. As before, we observed a greater than 3 -fold depletion of rRNA across the range of input starting material, including at the lowest starting amount of $0.02 \mathrm{ng}$ total RNA, with greater than $77 \%$ of the reads in the sequencing library deriving from mRNA molecules (Fig. 3a). Similarly, we observed that the total number of genes detected is higher than that in the control samples and is unaffected by the starting input amount of total RNA, except at the lower starting amounts of $0.2 \mathrm{ng}$ and 0.02 ng total RNA (Fig. 3b). Finally, we also observed that gene expression was highly correlated between different amounts of starting total RNA (Fig. 3c and Additional file 1: Fig. S6). These experiments conclusively demonstrate that we can successfully apply EMBR-seq to quantify gene expression from total RNA starting as low as $20 \mathrm{pg}$.

\section{rRNA depletion efficiency of EMBR-seq can be further improved through additional blocking primers}

In all the EMBR-seq experiments described above, we observed that $13-22 \%$ of the mapped reads still derived from rRNA and therefore, we next attempted to further improve the rRNA depletion efficiency of EMBR-seq. Analyzing the mapped coordinates of the rRNA-derived reads showed that while the $3^{\prime}$ blocking primers in 

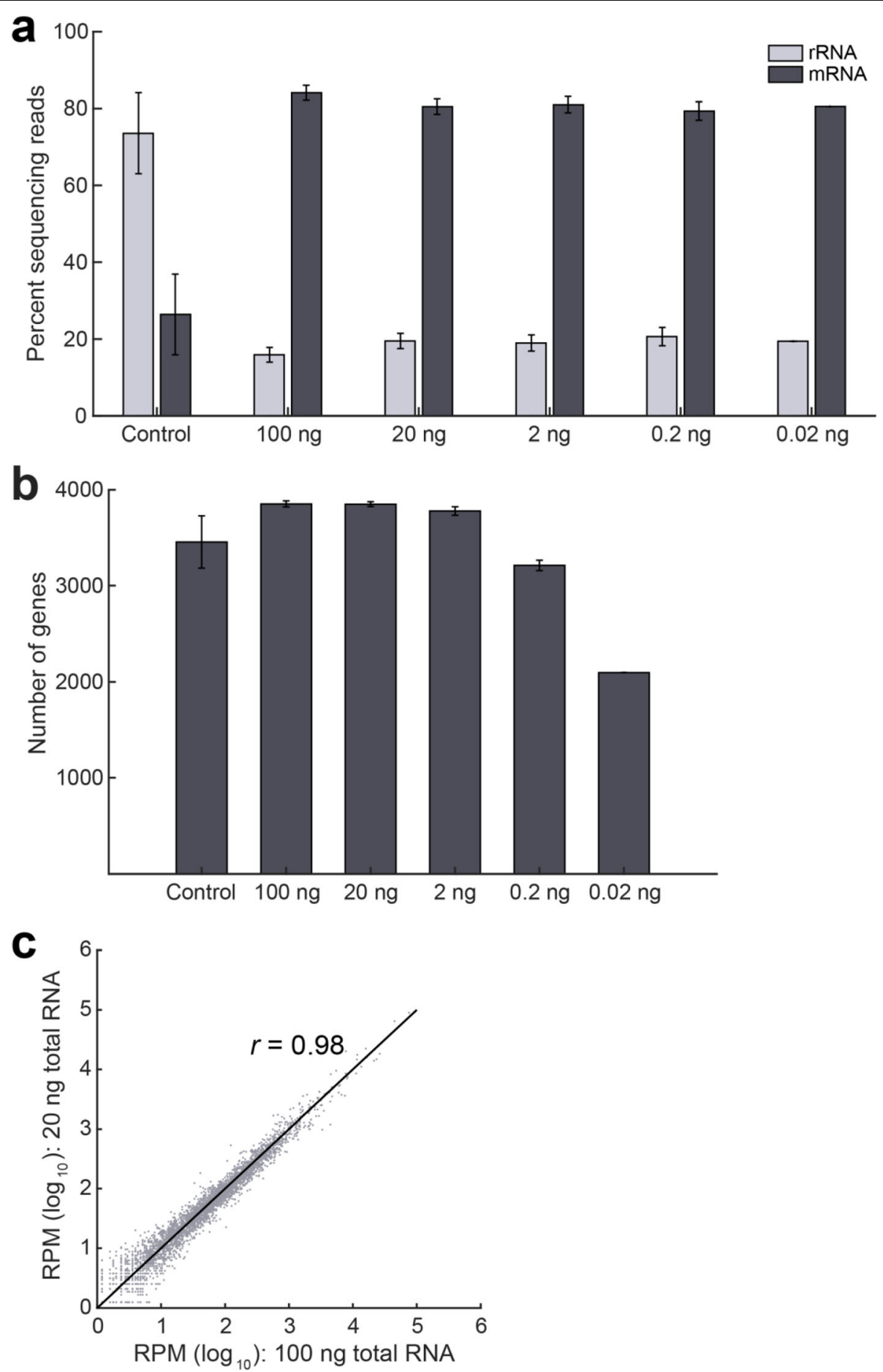

Fig. 3 EMBR-seq can quantify the transcriptome from low input total RNA. a Similar levels of rRNA depletion and mRNA enrichment are observed when the starting amount of total RNA is decreased from $100 \mathrm{ng}$ to $0.02 \mathrm{ng}(n \geq 2$, except at $0.02 \mathrm{ng}$ where $n=1)$. The control represents average data of control samples made from different input levels of total RNA. The $100 \mathrm{ng}$ data is reproduced from Fig. 2a. $\mathbf{b}$ Compared to the control samples, more genes are detected when starting with at least $2 \mathrm{ng}$ input total RNA. Fewer genes are detected when starting total RNA decreases to $0.02 \mathrm{ng}$. c Gene transcript counts are highly correlated (Pearson $r=0.98$ ) between $100 \mathrm{ng}$ and $20 \mathrm{ng}$ input total RNA in EMBR-seq. Datasets from lower starting total RNA are also well correlated to the $100 \mathrm{ng}$ samples (Additional file 1: Fig. S6) 
EMBR-seq effectively depleted rRNA-derived reads compared to control samples from the 3' end of rRNA molecules, specific "hotspot" regions along the entire length of the $16 \mathrm{~S}$ and $23 \mathrm{~S}$ rRNA were disproportionately abundant in the rRNA capture profile (Fig. 4a, b). We hypothesized that these reads resulted from the combined effects of poly-adenylation of fragmented RNA and biased capture of IVT amplified RNA molecules by random hexamer primers during reverse transcription. This reverse transcription step is part of the final Illumina library preparation protocol where the IVT amplified RNA is first reverse transcribed prior to generation of the Illumina libraries by PCR [20-22]. To minimize reads from these specific rRNA regions, we introduced 3 additional blocking primers per rRNA species that targeted the following hotspot locations: coordinates 107, 682,1241 on $16 \mathrm{~S}$ rRNA and coordinates $375,1421,1641$ on $23 \mathrm{~S}$ rRNA. We found that these hotspot blocking primers successfully reduced rRNA-derived reads from their target locations in the final sequencing library (Fig. $4 a, b)$. Overall, this resulted in further improvement in the rRNA depletion efficiency of EMBR-seq with only $10 \%$ of the mapped reads deriving from rRNA (Fig. 4c). These results demonstrate that EMBR-seq is a versatile technique that can be used to effectively deplete rRNA and can potentially be extended to target and deplete any undesired RNA species.

\section{Discussion}

We have developed a new technology, EMBR-seq, to efficiently deplete rRNA from total RNA, thereby enabling a deeper view of the genome-wide distribution of mRNA in bacterial samples. Sequencing bacterial mRNA poses several challenges; for example, the inability to easily enrich mRNA that typically makes up less than $5 \%$ of total RNA and the limiting starting amounts of total RNA that may be available when working with non-cultivable bacterial samples [7-9]. Through the use of a single 3' blocking primer per rRNA species, EMBR-seq efficiently minimizes the downstream amplification of rRNA molecules, thereby enabling a 4-fold depletion of rRNA in the final sequencing library (Figs. 1 and 2a). In the future, a

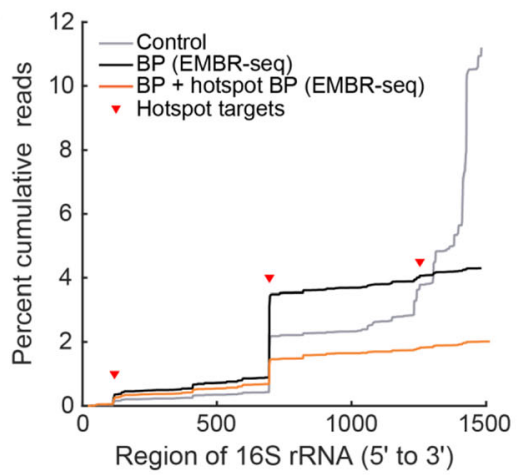

C

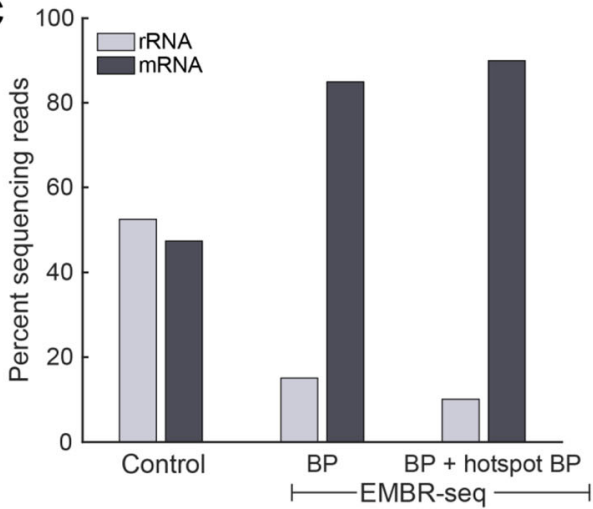

b

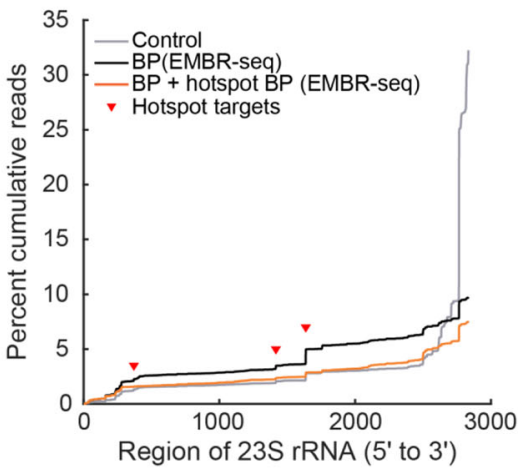

Fig. 4 Additional hotspot blocking primers increase the rRNA depletion efficiency of EMBR-seq. $\mathbf{a}$, $\mathbf{b}$ Cumulative percentage of sequencing reads ordered by mapping location along the (a) 165 and (b) 235 rRNA subunits, from 5' to $3^{\prime}$ ' ends of the transcript. In the control group, the majority of mapped reads are derived from the $3^{\prime}$ end together with a few "hotspot" locations (red triangles) along the gene body (gray lines). In EMBRseq, $3^{\prime}$ end blocking primers sharply reduce the number of reads derived from the $3^{\prime}$ end (black lines) with the remaining rRNA reads primarily deriving from hotspot locations. Additional blocking primers were designed to minimize poly-A tailing and amplification from the vicinity of these coordinates, resulting in further rRNA depletion (orange lines). $\mathbf{c}$ rRNA depletion and mRNA enrichment is enhanced upon the addition of hotspot blocking primers. With 3' end and hotspot blocking primers in EMBR-seq, mRNA molecules account for $90 \%$ of the mapped reads 
when introducing blocking primers at the $3^{\prime}$ end of an rRNA species, we hypothesize that the rRNA depletion efficiency could be further improved by designing primers with a $5^{\prime}$ overhang or a bulky $5^{\prime}$ modification. As demonstrated in this work, the design of blocking primers at the $3^{\prime}$ end of rRNA molecules efficiently depletes rRNA from high quality total RNA samples; however, certain practical applications can produce degraded and fragmented RNA in which rRNA molecules may be less effectively depleted. While we show that the 3' blocking primer alone is sufficient to obtain efficient rRNA depletion when starting with degraded E. coli total RNA, a more generalized strategy to overcome this challenge in EMBR-seq is to design additional blocking primers per rRNA species, that span the transcript length to minimize amplification of degraded rRNA molecules (Fig. 4 and Additional file 1: Fig. S1).

Starting with total RNA from E. coli, we show that efficient depletion of rRNA by EMBR-seq provides higher coverage of the transcriptome at the same sequencing depth (Fig. 2b and Additional file 1: Fig. S4). For example, compared to the control samples, the number of unique genes detected increases from 3628 to 3852 in EMBR-seq (Fig. 2b). In particular, EMBR-seq improves detection of lowly expressed genes below 500 RPM (Fig. $2 b)$. Further, EMBR-seq provides a more in-depth view of the transcriptional landscape without introducing technical artifacts. We find that $99 \%$ of the genes detected in the control group are also detected by EMBRseq, and that gene expression levels between the two groups are highly correlated (Fig. 2c, d).

As EMBR-seq typically uses a single blocking primer per rRNA species, sequence conservation analysis of $16 \mathrm{~S}$ and $23 \mathrm{~S}$ rRNA suggests that it is adaptable to other microbial species and complex bacterial communities (Additional file 1: Fig. S7). Recent approaches that employ a large array of probes also achieve a high efficiency of rRNA degradation; however, the need to generate such a large pool of molecules makes it more challenging to extrapolate these methods to evolutionarily distant bacterial species compared to EMBR-seq [16-18]. In addition, the use of just one or a few primers per rRNA species combined with the high level of sample multiplexing reduces cost significantly compared to other methods, enabling costeffective and high-throughput processing of hundreds of samples simultaneously (Additional file 1: Fig. S3 and Additional file 2). While the cost per rRNA depletion reaction of other reported techniques is an order-ofmagnitude higher than EMBR-seq, it is possible that the costs associated with these other methods and commercial kits could be reduced if all reagents and associated steps of the protocol are produced and performed in-house. Finally, beyond depleting rRNA and enriching for mRNA, the approach used in EMBR-seq can potentially also be used to target other high abundance transcripts in total RNA or used to enrich for non-coding RNA in both prokaryotic and eukaryotic systems [28-31].

We also demonstrated that EMBR-seq enables mRNA sequencing of low input RNA samples below the detection limit of commercial kits (Fig. 3a). Bacterial populations frequently contain diverse species, and even isogenic systems have been shown to display substantial cell-to-cell heterogeneity in gene expression that can give rise to dramatic cellular phenotypes [32-37]. Therefore, scaling down bacterial mRNA sequencing techniques to a singlecell level will enable quantification of this variability and provide a better understanding of how transcriptomic heterogeneity regulates cellular function $[38,39]$. Over the last few years, a limited number of approaches have been developed to sequence the transcriptome of single bacterial cells. Early proof-of-concept methods were low throughput techniques that sequenced less than 10 single cells and generally suffered from significant technical noise [10, 40, 41]. More recently, Blattman et al. employed combinatorial barcoding to circumvent single cell isolation, enabling high throughput single-cell sequencing of bacterial cell [42]. However, this method did not deplete rRNA, resulting in mRNA detection efficiencies of $\sim 2.5$ $10 \%$ (or $\sim 200$ mRNA per exponential phase $E$. coli cell). As an alternate approach, Imdahl et al. used MATQ-seq to generate sufficient cDNA from individually isolated bacterial cells; however, similar to the previous work, this method also did not deplete rRNA prior to sequencing [43]. In another study, Kuchina et al. combined rRNA depletion with combinatorial barcoding to achieve 5-10\% mRNA detection efficiencies in B. subtilis [14]. These initial efforts suggest that improved methods could significantly advance single-cell mRNA sequencing in bacteria. EMBR-seq can successfully sequence mRNA from as low as $20 \mathrm{pg}$ of total RNA; therefore, we anticipate that by coupling our rRNA depletion strategy with recent combinatorial barcoding techniques, we will be able to extend EMBR-seq to a single-cell resolution in the future [14, 42].

\section{Conclusion}

EMBR-seq efficiently depletes rRNA and provides a detailed view of the gene expression landscape within bacterial samples. As EMBR-seq depletes rRNA using a single or a few blocking primers per rRNA species, this new method is easily adaptable to other microbes as well as an order-of-magnitude cheaper than other reported techniques and commercial kits that frequently use a large array of probes to remove rRNA from total RNA. Finally, EMBR-seq effectively captures the transcriptome from 500-fold lower starting total RNA compared to commercial kits, thereby providing a powerful new approach to investigate gene expression patterns in rare and non-cultivable bacterial species. 


\section{Methods}

\section{Bacterial strains and culture conditions}

Escherichia coli MG1655 (ATCC: 700926) overnight cultures were inoculated into fresh LB medium at 1:50 and grown at $37^{\circ} \mathrm{C}$ with shaking $(150 \mathrm{rpm})$. Upon reaching the exponential growth phase, the culture was centrifuged at $3000 \mathrm{~g}$ for $10 \mathrm{~min}$. The media was removed and the pellet was resuspended in PBS to a concentration of $10^{7}$ cells per $\mu \mathrm{L}$. The cells were stored on ice and total RNA extraction was performed immediately.

\section{RNA extraction}

Trizol (Thermo Fisher Scientific, Cat. \# 15596018) RNA extraction was performed following the manufacturer's protocol. Briefly, $10^{8}$ cells were added to $750 \mu \mathrm{L}$ Trizol, mixed, and then combined with $150 \mu \mathrm{L}$ chloroform. After centrifugation, the clear aqueous layer was recovered and precipitated with $375 \mu \mathrm{L}$ of isopropanol and $0.67 \mu \mathrm{L}$ of GlycoBlue (Thermo Fisher Scientific, Cat. \# AM9515). The pellet was washed twice with $75 \%$ ethanol and after the final centrifugation, the resulting pellet was resuspended in RNase-free water.

\section{EMBR-seq}

\section{Poly adenylation}

$100 \mathrm{ng}$ of total RNA in $2 \mu \mathrm{L}$ was combined with $3 \mu \mathrm{L}$ poly-A mix, comprised of $1 \mu \mathrm{L} 5 \mathrm{x}$ first strand buffer [250 mM Tris-HCl (pH 8.3), $375 \mathrm{mM} \mathrm{KCl,} 15 \mathrm{mM}$ $\mathrm{MgCl}_{2}$, comes with Superscript II reverse transcriptase, Invitrogen Cat. \# 18064-014], $1 \mu \mathrm{L}$ blocking primer mix (see Primers), $0.8 \mu \mathrm{L}$ nuclease-free water, $0.1 \mu \mathrm{L} 10 \mathrm{mM}$ ATP, and $0.1 \mu \mathrm{L}$ E. coli poly-A polymerase (New England Biolabs, Cat. \# M0276S). The mixture was incubated at $37^{\circ} \mathrm{C}$ for $10 \mathrm{~min}$. In the control group, no blocking primers were added and $1.8 \mu \mathrm{L}$ of nuclease-free water was added instead. For EMBR-seq with either unmodified or phosphorylated 3'-end blocking primers, the blocking primer mix was prepared by mixing equal volumes of $50 \mu \mathrm{M}$ blocking primers specific to $5 \mathrm{~S}, 16 \mathrm{~S}$ and $23 \mathrm{~S}$ rRNA. For EMBR-seq with hotspot blocking primers, the blocking primer mix was prepared by mixing equal volumes of $100 \mu \mathrm{M} 3$ '-end blocking primers with $100 \mu \mathrm{M}$ hotspot blocking primers, such that the final mixture was $50 \mu \mathrm{M} 33^{\prime}$-end primers (3 primers mixed) and $50 \mu \mathrm{M}$ hotspot primers (6 primers mixed).

\section{Reverse transcription}

The polyadenylation product was mixed with $0.5 \mu \mathrm{L} 10$ mM dNTPs (New England Biolabs, Cat. \# N0447L), $1 \mu \mathrm{L}$ reverse transcription primers $(25 \mathrm{ng} / \mu \mathrm{L}$, see Primers $)$, and $1.3 \mu \mathrm{L}$ blocking primer mix, and heated to $65^{\circ} \mathrm{C}$ for $5 \mathrm{~min}, 58^{\circ} \mathrm{C}$ for $1 \mathrm{~min}$, and then quenched on ice. In the control samples, the blocking primers were again replaced with nuclease-free water. Next, 3.2 $\mu \mathrm{L}$ RT mix, consisting of $1.2 \mu \mathrm{L} 5 \mathrm{x}$ first strand buffer, $1 \mu \mathrm{L} 0.1 \mathrm{M}$ DTT, $0.5 \mu \mathrm{L}$ RNaseOUT (Thermo Fisher Scientific, Cat. \#10777019), and $0.5 \mu \mathrm{L}$ Superscript II reverse transcriptase was added to the solution, followed by $1 \mathrm{~h}$ incubation at $42{ }^{\circ} \mathrm{C}$. The temperature was then raised to $70{ }^{\circ} \mathrm{C}$ for $10 \mathrm{~min}$ to heat inactivate Superscript II.

\section{Second strand synthesis}

$49 \mu \mathrm{L}$ of the second strand mix, containing $33.5 \mu \mathrm{L}$ water, $12 \mu \mathrm{L} 5 \mathrm{x}$ second strand buffer $[100 \mathrm{mM}$ Tris- $\mathrm{HCl}(\mathrm{pH}$ 6.9), $23 \mathrm{mM} \mathrm{MgCl}_{2}, 450 \mathrm{mM} \mathrm{KCl}, 0.75 \mathrm{mM} \beta-\mathrm{NAD}, 50$ $\mathrm{mM}\left(\mathrm{NH}_{4}\right)_{2} \mathrm{SO}_{4}$, Invitrogen, Cat. \# 10812-014], $1.2 \mu \mathrm{L} 10$ $\mathrm{mM}$ dNTPs, $0.4 \mu \mathrm{L}$ E. coli ligase (Invitrogen, Cat. \# 18052-019), $1.5 \mu \mathrm{L}$ DNA polymerase I (Invitrogen, Cat. \# 18010-025), and $0.4 \mu \mathrm{L}$ RNase $\mathrm{H}$ (Invitrogen, Cat. \# 18021-071), was added to the product from the previous step. The mixture was incubated at $16^{\circ} \mathrm{C}$ for $2 \mathrm{~h}$. cDNA was purified with 1x AMPure XP DNA beads (Beckman Coulter, Cat. \# A63881) and eluted in $24 \mu \mathrm{L}$ nuclease-free water that was subsequently concentrated to $6.4 \mu \mathrm{L}$.

\section{In vitro transcription}

The concentrated solution was mixed with $9.6 \mu \mathrm{L}$ of Ambion in vitro transcription mix $(1.6 \mu \mathrm{L}$ of each ribonucleotide, $1.6 \mu \mathrm{L} 10 \mathrm{x}$ T7 reaction buffer, $1.6 \mu \mathrm{L}$ T7 enzyme mix, MEGAscript T7 Transcription Kit, Thermo Fisher Scientific, Cat. \# AMB13345) and incubated at $37^{\circ} \mathrm{C}$ for $13 \mathrm{~h}$. Next, the aRNA was treated with $6 \mu \mathrm{L}$ EXO-SAP (ExoSAP-IT ${ }^{\mathrm{Ts}}$ PCR Product Cleanup Reagent, Thermo Fisher Scientific, Cat. \# 78200.200.UL) at $37^{\circ} \mathrm{C}$ for $15 \mathrm{~min}$ followed by fragmentation with $5.5 \mu \mathrm{L}$ fragmentation buffer (200 mM Tris-acetate (pH 8.1), 500 $\mathrm{mM} \mathrm{KOAc,} 150 \mathrm{mM} \mathrm{MgOAc}$ ) at $94{ }^{\circ} \mathrm{C}$ for $3 \mathrm{~min}$. The reaction was then quenched with $2.75 \mu \mathrm{L}$ stop buffer (0.5 M EDTA) on ice. The fragmented aRNA was size selected with 0.8x AMPure RNA beads (RNAClean XP Kit, Beckman Coulter, Cat. \# A63987) and eluted in $15 \mu \mathrm{L}$ nuclease-free water. Thereafter, Illumina libraries were prepared as described previously [20].

\section{EMBR-seq with TEX digestion}

To test the Terminator ${ }^{\mathrm{rm}} 5^{\prime}$-phosphate-dependent exonuclease (Lucigen, Cat. \# TER5120), 100 ng of total RNA in $2 \mu \mathrm{L}$ was combined with $18 \mu \mathrm{L}$ TEX mix, comprised of $14.5 \mu \mathrm{L}$ nuclease free water, $2 \mu \mathrm{L}$ Terminator $10 \mathrm{x}$ buffer $\mathrm{A}, 0.5 \mu \mathrm{L}$ RNAseOUT, and $1 \mu \mathrm{L}$ TEX. The solution was incubated at $30^{\circ} \mathrm{C}$ for $1 \mathrm{~h}$ and quenched with $1 \mu \mathrm{L}$ of $100 \mathrm{mM}$ EDTA. The product was purified with $1 \mathrm{x}$ AMPure RNA beads and eluted in $10 \mu \mathrm{L}$ nuclease-free water and concentrated to $2 \mu \mathrm{L}$. This TEX digested total RNA was then used as starting RNA in the EMBR-seq protocol described above. 


\section{EMBR-seq bioinformatic analysis}

Paired-end sequencing of the EMBR-seq libraries was performed on an Illumina NextSeq 500. All sequencing data has been deposited to Gene Expression Omnibus under the accession number GSE149666. In the sequencing libraries, the left mate contains information about the sample barcode (see Primers). The right mate is mapped to the bacterial transcriptome. Prior to mapping, only reads containing valid sample barcodes were retained. Subsequently, the reads were mapped to the reference transcriptome ( $E$. coli K12 substr. MG1655 cds ASM584v2) using BurrowsWheeler Aligner (BWA) with default parameters.

\section{Analysis of detection bias in EMBR-seq}

E. coli operons were downloaded from RegulonDB [44]. Operons with at least 2 genes were included for this analysis. The data from EMBR-seq libraries with $100 \mathrm{ng}$ starting material was mapped to E. coli K12 substr. MG1655 reference genome (ASM584v2). For each read that maps within an operon, the distance of the mapped location from the 3' end of the operon was calculated, accounting for the read length. Next, the operons were discretized into 50 bins, and all operons with more than 200 unique reads were considered for downstream analysis. The number of reads in each bin was then normalized by the total number of reads in each operon, and the average of the relative reads within each bin was calculated. To compare bacterial data from EMBR-seq to mammalian data from CEL-seq, we downloaded CEL-seq data reported in Grün et al. (GEO Accession: GSM1322290) and performed similar analysis for the mouse genes [45].

\section{Sequence conservation of $16 \mathrm{~S}$ and $23 \mathrm{~S}$ rRNA}

16S rRNA sequences from 4000 species were obtained from $r r n D B$ [46], while 23S rRNA sequences from 119 species were selected from NCBI RefSeq [47]. Next, the last 100 bases from the 3'end of each sequence were aligned using Clustal Omega [48]. Shannon entropy for each aligned base location was then calculated such that the maximal entropy value was 1 . Five possibilities were allowed: "A", "T", "C", "G", and “-".

\section{Primers}

Reverse transcription primers are shown below with the 6-nucleotide sample barcodes underlined [20]:

GCCGGTAATACGACTCACTATAGGGAGTTCTA CAGTCCGACGATCNNNNNN(NNNN NN)TTTTTTTTTTTTTTTTTTTTTTTTV

The following five barcodes were used in this study:

AGACTC

$\underline{\text { AGCTTC }}$

CATGAG

CAGATC

TCACAG
Blocking primers:

5S 5'-ATGCCTGGCAGTTCCCTACTCTCGCA TGGG-3'

$16 \mathrm{~S} 5$ '-TAAGGAGGTGATCCAACCGCAGGTTC CCCT-3'

23S 5'-AAGGTTAAGCCTCACGGTTCATTAGT ACCG-3'

In the case of the 3' phosphorylated primers, all blocking primers have a 3 ' phosphorylation modification.

Hotspot blocking primers:

16S primer for hotspot at position 107:

5'-GGCACATCCGATGGCAAGAGGCCCGAAGGT-3' . $16 \mathrm{~S}$ primer for hotspot at position 682 :

5' -TCCTGTTTGCTCCCCACGCTTTCGCACCTG-3' .

16S primer for hotspot at position 1241:

5'-CCGTGGCATTCTGATCCACGATTACTAGCG ATTCCG-3.

23S primer for hotspot at position 375:

5'-CGCCTTTCCCTCACGGTACTGGTTCACTAT

CGG-3'.

23S primer for hotspot at position 1421:

5'-TTGCTTCAGCACCGTAGTGCCTCGTCATCA-3'.

23S primer for hotspot at position 1641:

5'-GCAGCCAGCTGGTATCTTCGACTGATTTCA GC-3'.

Each primer is designed to anneal approximately 100 bp downstream of the hotspot. The exact position and length of each primer was adjusted to ensure the $T_{m}$ was above $65^{\circ} \mathrm{C}$.

\section{Supplementary information}

Supplementary information accompanies this paper at https://doi.org/10. 1186/s12864-020-07134-4.

Additional file 1 Figure S1. EMBR-seq effectively depletes rRNA from fragmented total RNA. Figure S2. Combining TerminatorTM 5'-phosphatedependent exonuclease (TEX) digestion with EMBR-seq does not improve rRNA depletion. Figure S3. Cost associated with performing EMBR-seq. Figure S4. Higher number of genes detected in EMBR-seq is not dependent on the sequencing depth. Figure S5. Distribution of reads along E. coli operons in EMBR-seq. Figure S6. Gene transcript count correlation between different input total RNA amounts in EMBR-seq. Figure S7. Quantification of 165 and 235 rRNA sequence conservation using Shannon entropy. Table S1. Comparison of rRNA depletion methods.

Additional file 2.

\section{Abbreviations}

IVT: In vitro transcription; LB: Lysogeny broth; RNase $\mathrm{H}$ : Ribonuclease $\mathrm{H}$; TEX: Terminator ${ }^{\mathrm{TM}}$ exonuclease

\section{Acknowledgements}

We would like to thank members of the Dey and O'Malley groups for helpful discussions. The authors thank Dr. Jennifer Smith for assistance with sequencing Illumina libraries. Sequencing was performed at the Biological Nanostructures Laboratory within the California NanoSystems Institute (CNSI), supported by the University of California, Santa Barbara (UCSB) and the University of California, Office of the President (UCOP). We further acknowledge the Center of Scientific Computing at UCSB for computational facilities that are funded by NSF MRSEC (DMR-1720256) and NSF CNS1725797. 


\section{Authors' contributions}

C.W. and S.S.D. conceived the method, C.W., K.A.H. and S.S.D. designed the experiments. K.A.H., C.W. and E.L. participated in data collection. K.A.H. and C.W. analyzed the data. C.W., K.A.H., M.A.O. and S.S.D. wrote the manuscript S.S.D. guided experimental design and data analysis. All authors have read and approved the final version of the manuscript.

\section{Funding}

This work was funded by grants from the National Science Foundation (MCB-1553721) to M.A.O., the UCSB Academic Senate Faculty Research Grant to S.S.D., and the CNSI Challenge Grant Program, supported by UCSB and UCOP, to S.S.D. and M.A.O.

\section{Availability of data and materials}

The datasets generated and analyzed in the current study have been deposited to GEO under the accession number GSE149666.

\section{Ethics approval and consent to participate}

Not applicable.

\section{Consent for publication}

Not applicable.

\section{Competing interests}

The authors declare no competing financial interests.

\section{Author details}

'Department of Chemical Engineering, University of California Santa Barbara, Santa Barbara, CA 93106, USA. ${ }^{2}$ Center for Bioengineering, University of California Santa Barbara, Santa Barbara, CA 93106, USA. ${ }^{3}$ Neuroscience Research Institute, University of California Santa Barbara, Santa Barbara, CA 93106, USA.

Received: 21 May 2020 Accepted: 9 October 2020

Published online: 16 October 2020

\section{References}

1. Otero JM, Nielsen J. Industrial systems biology. Biotechnol Bioeng. 2010;105: 439-60

2. Dvořák P, Nikel PI, Damborský J, de Lorenzo V. Bioremediation 3.0: engineering pollutant-removing bacteria in the times of systemic biology. Biotechnol Adv. 2017;35:845-66.

3. Peng X, Gilmore SP, O'Malley MA. Microbial communities for bioprocessing: lessons learned from nature. Curr Opion Chem Eng. 2016;14:103-9.

4. Kung Y, Runguphan W, Keasling JD. From fields to fuels: recent advances in the microbial production of biofuels. ACS Synth Biol. 2012;1:498-513.

5. Barajas JF, Blake-Hedges JM, Bailey CB, Curran S, Keasling JD. Engineered polyketides: synergy between protein and host level engineering. Synth Syst Biotechnol. 2017:2:147-66.

6. Creecy JP, Conway T. Quantitative bacterial transcriptomics with RNA-seq. Curr Opin Microbiol. 2015;23:133-40.

7. Mortazavi A, Williams BA, McCue K, Schaeffer L, Wold B. Mapping and quantifying mammalian transcriptomes by RNA-Seq. Nat Methods. 2008;5: 621-8.

8. Proudfoot NJ. Ending the message: poly(A) signals then and now. Genes Dev. 2011:25:1770-82

9. Giannoukos G, Ciulla DM, Huang K, Haas BJ, Izard J, Levin JZ, et al. Efficient and robust RNA-seq process for cultured bacteria and complex community transcriptomes. Genome Biol. 2012;13:r23.

10. Kang Y, Norris MH, Zarzycki-Siek J, Nierman WC, Donachie SP, Hoang TT. Transcript amplification from single bacterium for transcriptome analysis. Genome Res. 2011;21:925-35.

11. Petrova OE, Garcia-Alcalde F, Zampaloni C, Sauer K. Comparative evaluation of rRNA depletion procedures for the improved analysis of bacterial biofilm and mixed pathogen culture transcriptomes. Sci Rep. 2017:7:41114.

12. Kraus AJ, Brink BG, Siegel TN. Efficient and specific oligo-based depletion of rRNA. Sci Rep. 2019:9:12281.

13. Culviner PH, Guegler CK, Laub MT. A simple, cost-effective, and robust method for rRNA depletion in RNA-sequencing studies. mBio. 2020;11: e00010-20.
14. Kuchina A, Brettner LM, Paleologu L, Roco CM, Rosenberg AB, Carignano A, et al. Microbial single-cell RNA sequencing by split-pool barcoding. Preprint. bioRxiv. 2019. https://doi.org/10.1101/869248.

15. He S, Wurtzel O, Singh K, Froula JL, Yilmaz S, Tringe SG, et al. Validation of two ribosomal RNA removal methods for microbial metatranscriptomics. Nat Methods. 2010;7:807-12.

16. Huang Y, Sheth RU, Kaufman A, Wang HH. Scalable and cost-effective ribonuclease-based rRNA depletion for transcriptomics. Nucleic Acids Res. 2020;48:e20.

17. Prezza G, Heckel T, Dietrich S, Homberger C, Westermann AJ, Vogel J. Improved bacterial RNA-seq by Cas9-based depletion of ribosomal RNA reads. RNA. 2020;26:1069-78.

18. Armour CD, Castle JC, Chen R, Babak T, Loerch P, Jackson S, et al. Digita transcriptome profiling using selective hexamer priming for CDNA synthesis. Nat Methods. 2009:6:647-9.

19. Feng Y, Cohen SN. Unpaired terminal nucleotides and $5^{\prime}$ monophosphorylation govern 3' polyadenylation by Escherichia coli poly(A) polymerase I. Proc Natl Acad Sci U S A. 2000;97:6415-20.

20. Hashimshony T, Senderovich N, Avital G, Klochendler A, de Leeuw Y, Anavy $L$, et al. CEL-Seq2: sensitive highly-multiplexed single-cell RNA-Seq. Genome Biol. 2016:17:77.

21. Mooijman D, Dey SS, Boisset J-C, Crosetto N, van Oudenaarden A. Singlecell $5 \mathrm{hmC}$ sequencing reveals chromosome-wide cell-to-cell variability and enables lineage reconstruction. Nat Biotechnol. 2016;34:852-6.

22. Rooijers K, Markodimitraki CM, Rang FJ, de Vries SS, Chialastri A, de Luca KL, et al. Simultaneous quantification of protein-DNA contacts and transcriptomes in single cells. Nat Biotechnol. 2019;37:766-72.

23. Wendisch VF, Zimmer DP, Khodursky A, Peter B, Cozzarelli N, Kustu S. Isolation of Escherichia coli mRNA and comparison of expression using mRNA and total RNA on DNA microarrays. Anal Biochem. 2001:290:205-13.

24. Westermann AJ, Förstner KU, Amman F, Barquist L, Chao Y, Schulte LN, et al. Dual RNA-seq unveils noncoding RNA functions in host-pathogen interactions. Nature. 2016;529:496-501.

25. Westermann AJ, Gorski SA, Vogel J. Dual RNA-seq of pathogen and host. Nat Rev Microbiol. 2012;10:618-30.

26. Cozen AE, Quartley E, Holmes AD, Hrabeta-Robinson E, Phizicky EM, Lowe TM. ARM-seq: AlkB-facilitated RNA methylation sequencing reveals a complex landscape of modified tRNA fragments. Nat Methods. 2015;12:87984.

27. Zheng G, Qin Y, Clark WC, Dai Q, Yi C, He C, et al. Efficient and quantitative high-throughput tRNA sequencing. Nat Methods. 2015;12:835-7.

28. Sharma CM, Hoffmann S, Darfeuille F, Reignier J, Findeiss S, Sittka A, et al. The primary transcriptome of the major human pathogen Helicobacter pylori. Nature. 2010;464:250-5.

29. Chao Y, Papenfort K, Reinhardt R, Sharma CM, Vogel J. An atlas of Hfabound transcripts reveals $3^{\prime}$ UTRs as a genomic reservoir of regulatory small RNAs: Hfq-dependent small RNAs from 3' UTRs. EMBO J. 2012:31:4005-19.

30. Chao Y, Li L, Girodat D, Förstner KU, Said N, Corcoran C, et al. In vivo cleavage map illuminates the central role of RNase $\mathrm{E}$ in coding and noncoding RNA pathways. Mol Cell. 2017;65:39-51.

31. Faridani OR, Abdullayev I, Hagemann-Jensen M, Schell JP, Lanner F, Sandberg R. Single-cell sequencing of the small-RNA transcriptome. Nat Biotechnol. 2016:34:1264-6.

32. Miller-Jensen K, Dey SS, Schaffer DV, Arkin AP. Varying virulence: epigenetic control of expression noise and disease processes. Trends Biotechnol. 2011; 29:517-25.

33. Raj A, van Oudenaarden A. Nature, nurture, or chance: stochastic gene expression and its consequences. Cell. 2008;135:216-26.

34. Balázsi G, van Oudenaarden A, Collins JJ. Cellular decision making and biological noise: from microbes to mammals. Cell. 2011;144:910-25.

35. Gefen $\mathrm{O}$, Balaban NQ. The importance of being persistent: heterogeneity of bacterial populations under antibiotic stress. FEMS Microbiol Rev. 2009:33: 704-17.

36. Russell JR, Cabeen MT, Wiggins PA, Paulsson J, Losick R. Noise in a phosphorelay drives stochastic entry into sporulation in Bacillus subtilis. EMBO J. 2017:36:2856-69.

37. Avraham R, Haseley N, Brown D, Penaranda C, Jijon HB, Trombetta JJ, et al Pathogen cell-to-cell variability drives heterogeneity in host immune responses. Cell. 2015;163:523.

38. Penaranda C, Hung DT. Single-cell RNA sequencing to understand hostpathogen interactions. ACS Infect Dis. 2019;5:336-44. 
39. Avital G, Avraham R, Fan A, Hashimshony T, Hung DT, Yanai I. scDual-Seq: mapping the gene regulatory program of Salmonella infection by host and pathogen single-cell RNA-sequencing. Genome Biol. 2017;18:200.

40. Wang J, Chen L, Chen Z, Zhang W. RNA-seq based transcriptomic analysis of single bacterial cells. Integr Biol (Camb). 2015;7:1466-76.

41. Liu Y, Jeraldo P, Jang JS, Eckloff B, Jen J, Walther-Antonio M. Bacterial single cell whole transcriptome amplification in microfluidic platform shows putative gene expression heterogeneity. Anal Chem. 2019;91:8036-44.

42. Blattman SB, Jiang W, Oikonomou P, Tavazoie S. Prokaryotic single-cell RNA sequencing by in situ combinatorial indexing. Nat Microbiol. 2020. https:// doi.org/10.1038/s41564-020-0729-6

43. Imdahl F, Vafadarnejad E, Homberger C, Saliba AE, Vogel J. Single-cell RNAsequencing reports growth-condition-specific global transcriptomes of individual bacteria. Nat Microbiol. 2020. https://doi.org/10.1038/s41564-0200774-1.

44. Santos-Zavaleta A, Salgado H, Gama-Castro S, Sánchez-Pérez M, GómezRomero L, Ledezma-Tejeida D, et al. RegulonDB v 10.5: tackling challenges to unify classic and high throughput knowledge of gene regulation in $E$. coli K-12. Nucleic Acids Res. 2019;47:D212-20.

45. Grün D, Kester L, van Oudenaarden A. Validation of noise models for singlecell transcriptomics. Nat Methods. 2014;11:637-40.

46. Stoddard SF, Smith BJ, Hein R, BRK R, Schmidt TM. rrnDB: improved tools for interpreting rRNA gene abundance in bacteria and archaea and a new foundation for future development. Nucleic Acids Res. 2015;43:D593-8.

47. O'Leary NA, Wright MW, Brister JR, Ciufo S, Haddad D, McVeigh R, et al. Reference sequence (RefSeq) database at NCBI: current status, taxonomic expansion, and functional annotation. Nucleic Acids Res. 2016;44:D733-45.

48. Madeira F, Park YM, Lee J, Buso N, Gur T, Madhusoodanan N, et al. The EMBL-EBI search and sequence analysis tools APIs in 2019. Nucleic Acids Res. 2019;47:W636-41.

\section{Publisher's Note}

Springer Nature remains neutral with regard to jurisdictional claims in published maps and institutional affiliations.

Ready to submit your research? Choose BMC and benefit from:

- fast, convenient online submission

- thorough peer review by experienced researchers in your field

- rapid publication on acceptance

- support for research data, including large and complex data types

- gold Open Access which fosters wider collaboration and increased citations

- maximum visibility for your research: over $100 \mathrm{M}$ website views per year

At $\mathrm{BMC}$, research is always in progress.

Learn more biomedcentral.com/submissions 